\title{
Direct Two-Point Block One-Step Method for Solving General Second-Order Ordinary Differential Equations
}

\author{
Zanariah Abdul Majid, ${ }^{1,2}$ Nur Zahidah Mokhtar, ${ }^{1}$ \\ and Mohamed Suleiman ${ }^{1,2}$ \\ ${ }^{1}$ Institute for Mathematical Research, Universiti Putra Malaysia, Selangor Darul Ehsan, \\ 43400 Serdang, Malaysia \\ ${ }^{2}$ Mathematics Department, Faculty of Science, Universiti Putra Malaysia, Selangor Darul Ehsan, \\ 43400 Serdang, Malaysia
}

Correspondence should be addressed to Zanariah Abdul Majid, zanariah@math.upm.edu.my

Received 25 May 2011; Revised 19 September 2011; Accepted 30 September 2011

Academic Editor: Gerhard-Wilhelm Weber

Copyright (c) 2012 Zanariah Abdul Majid et al. This is an open access article distributed under the Creative Commons Attribution License, which permits unrestricted use, distribution, and reproduction in any medium, provided the original work is properly cited.

\begin{abstract}
A direct two-point block one-step method for solving general second-order ordinary differential equations (ODEs) directly is presented in this paper. The one-step block method will solve the second-order ODEs without reducing to first-order equations. The direct solutions of the general second-order ODEs will be calculated at two points simultaneously using variable step size. The method is formulated using the linear multistep method, but the new method possesses the desirable feature of the one-step method. The implementation is based on the predictor and corrector formulas in the $P E(C E)^{m}$ mode. The stability and precision of this method will also be analyzed and deliberated. Numerical results are given to show the efficiency of the proposed method and will be compared with the existing method.
\end{abstract}

\section{Introduction}

In this paper, we are considering solving directly the general second-order initial value problems (IVPs) for systems of ODEs in the form

$$
y^{\prime \prime}=f\left(x, y, y^{\prime}\right), \quad y(a)=y_{0}, \quad y^{\prime}(a)=y_{0}^{\prime}, \quad x \in[a, b]
$$

Equation in (1.1) arises from many physical phenomena in a wide spectrum of applications especially in the science and engineering areas such as in the electric circuit, damped and 


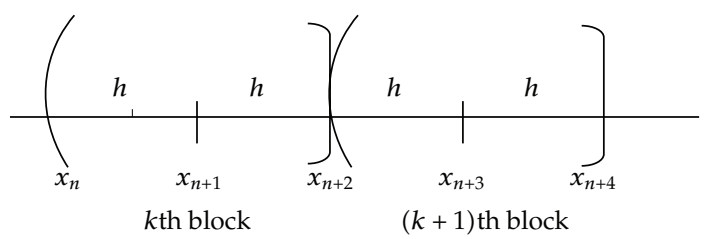

Figure 1: Block one-step method.

undamped spring mass and some other areas of application. We will also consider solving general second order as in (1.1) using the direct block one-step method. Block methods for numerical solutions of ODEs have been proposed by several researchers such as in [1-5]. The common block methods used to solve the problems can be categorized as one-step block method and multistep block method.

One-step block method such as the implicit Runge-Kutta method is also being referred to as one previous point to obtain the solution. The multistep block method in the form of Adams type formula is presented in [5, 6]. In [7], the block backward differentiation formula (BBDF) for solving stiff ODEs has been introduced and the solutions referred to as more than one previous point. The works in [6] showed the proposed two-point four-step block method presented as in a simple form of Adams Moulton method for solving second-order ODEs directly.

The block method of Runge-Kutta type has been explored in [1], and it is suggested that a block of new approximation values is used simultaneously for solving first-order ODEs. The works in $[3,8,9]$ have been considered in solving (1.1) using the block one-step method, while [3] has proposed a two-point implicit block one-step method for solving second-order ODEs directly and suggested that the method is suitable to be parallel.

In [9], Majid et al. have derived the two-point block method for solving first-order ODEs by using the closest point in the interval, that is, $\left[x_{n}, x_{n+1}\right]$ and $\left[x_{n+1}, x_{n+2}\right]$. The Gauss Seidel iteration was implemented in the proposed block method. The approach in this research is to extend the idea in [9] for solving (1.1) directly without reducing system of first-order ODEs using two-point block one-step method.

\section{Formulation of the Method}

In order to compute the two approximation values of $y_{n+1}$ and $y_{n+2}$ simultaneously, the interval of $[a, b]$ is divided into a series of blocks with each block containing two points as shown in Figure 1.

In Figure 1, we observed that $k$ th block contains $x_{n}, x_{n+1}$ and $x_{n+2}$, where $x_{n}$ becomes the starting point and $x_{n+2}$ is the last point in the $k$ th block with step size $h$. The approximations values of $y_{n+1}$ and $y_{n+2}$ are computed simultaneously. The evaluation solution at the last point in $k$ th block will be restored as the initial values for $(k+1)$ th block. The same procedure is used to compute the solutions for the next block until the end of the interval. The evaluation information from the previous step in a block could be used for other steps of the same block only. During the calculations of iteration, the final values of $y_{n+2}$ at the point $x_{n+2}$ are taken as the initial values for the next iteration. 
We obtained the approximation values of $y_{n+1}$ and $y_{n+2}$ at the points $x_{n+1}$ and $x_{n+2}$ by integrating once and twice over (1.1) with respect to $x$. We begin to evaluate the $y_{n+1}^{\prime}$ and $y_{n+1}$ by integrating once and twice (1.1) over the interval $\left[x_{n}, x_{n+1}\right]$ :

$$
\begin{aligned}
\int_{x_{n}}^{x_{n+1}} y^{\prime \prime}(x) d x & =\int_{x_{n}}^{x_{n+1}} f\left(x, y, y^{\prime}\right) d x \\
\int_{x_{n}}^{x_{n+1}} \int_{x_{n}}^{x} y^{\prime \prime}(x) d x d x & =\int_{x_{n}}^{x_{n+1}} \int_{x_{n}}^{x} f\left(x, y, y^{\prime}\right) d x d x .
\end{aligned}
$$

Let $x_{n+1}=x_{n}+h$ which gives

$$
\begin{gathered}
y^{\prime}\left(x_{n+1}\right)-y^{\prime}\left(x_{n}\right)=\int_{x_{n}}^{x_{n+1}} f\left(x, y, y^{\prime}\right) d x \\
y\left(x_{n+1}\right)-y\left(x_{n}\right)-h y^{\prime}\left(x_{n}\right)=\int_{x_{n}}^{x_{n+1}}\left(x_{n+1}-x\right) f\left(x, y, y^{\prime}\right) d x .
\end{gathered}
$$

Then, $f\left(x, y, y^{\prime}\right)$ in (2.2) will be replaced with Lagrange interpolation polynomial that involves interpolation points in the block at $\left(x_{n}, f_{n}\right),\left(x_{n+1}, f_{n+1}\right)$ and $\left(x_{n+2}, f_{n+2}\right)$. Taking $x=x_{n+2}+\operatorname{sh}, s=\left(x-x_{n+2}\right) / h$, and $d x=h d s$ and replacing into (2.2). Then, the limit of integration in (2.2) will be from -2 to -1 . The corrector formulae will be obtained using MATHEMATICA. The formulae of $y_{n+1}^{\prime}$ and $y_{n+1}$ are obtained as follows:

$$
\begin{gathered}
y_{n+1}^{\prime}=y_{n}^{\prime}+\frac{h}{12}\left(5 f_{n}+8 f_{n+1}-f_{n+2}\right), \\
y_{n+1}=y_{n}+h y_{n}^{\prime}+\frac{h^{2}}{24}\left(7 f_{n}+6 f_{n+1}-f_{n+2}\right) .
\end{gathered}
$$

To approximate the value of $y_{n+2}^{\prime}$ and $y_{n+2}$, we take $x_{n+2}=x_{n}+2 h$ by integrating once and twice (1.1) over the interval $\left[x_{n+1}, x_{n+2}\right]$ and apply the same process. The limit of integration will be from -1 to 0 , and the following corrector formulae will be obtained:

$$
\begin{gathered}
y_{n+2}^{\prime}=y_{n+1}^{\prime}+\frac{h}{12}\left(-f_{n}+8 f_{n+1}+5 f_{n+2}\right), \\
y_{n+2}=y_{n+1}+h y_{n+1}^{\prime}+\frac{h^{2}}{24}\left(-f_{n}+10 f_{n+1}+3 f_{n+2}\right) .
\end{gathered}
$$


The formulae (2.3) and (2.4) may be rewritten in the form of matrix difference equation as follows:

$$
\left[\begin{array}{cccc}
0 & 0 & 0 & 0 \\
0 & -1 & 1 & 0 \\
0 & 0 & 0 & 0 \\
0 & 0 & -1 & 1
\end{array}\right]\left[\begin{array}{c}
y_{n-1} \\
y_{n} \\
y_{n+1} \\
y_{n+2}
\end{array}\right]=h\left(\left[\begin{array}{cccc}
0 & 1 & -1 & 0 \\
0 & 1 & 0 & 0 \\
0 & 0 & 1 & -1 \\
0 & 0 & 1 & 0
\end{array}\right]\left[\begin{array}{c}
y_{n-1}^{\prime} \\
y_{n}^{\prime} \\
y_{n+1}^{\prime} \\
y_{n+2}^{\prime}
\end{array}\right]\right)+h^{2}\left(\left[\begin{array}{cccc}
0 & \frac{5}{12} & \frac{8}{12} & \frac{-1}{12} \\
0 & \frac{7}{24} & \frac{6}{24} & \frac{-1}{24} \\
0 & \frac{-1}{12} & \frac{8}{12} & \frac{5}{12} \\
0 & \frac{-1}{24} & \frac{10}{24} & \frac{3}{24}
\end{array}\right]\left[\begin{array}{c}
f_{n-1} \\
f_{n} \\
f_{n+1} \\
f_{n+2}
\end{array}\right]\right) .
$$

The order of this developed method is identified by referring to [10-12]. The two-point onestep block method for ODEs can be written in a matrix difference equation as follows:

$$
\alpha Y_{m}=h \beta Y_{m}^{\prime}+h^{2} \gamma F_{m}
$$

where $\alpha, \beta$, and $\gamma$ are the coefficients with the $m$-vector $Y_{m}, Y_{m}^{\prime}$, and $F_{m}$ be defined as

$$
Y_{m}=\left[y_{n-1}, y_{n}, y_{n+1}, y_{n+2}\right]^{T}, \quad Y_{m}^{\prime}=\left[y_{n-1}^{\prime}, y_{n}^{\prime}, y_{n+1}^{\prime}, y_{n+2}^{\prime}\right]^{T}, \quad F_{m}=\left[f_{n-1}, f_{n}, f_{n+1}, f_{n+2}\right]^{T} .
$$

By applying the formulae for the constants $C_{q}$, in [10] the formulae is defined as

$$
\begin{aligned}
C_{0} & =\sum_{j=0}^{k} \alpha_{j}, \\
C_{1} & =\sum_{j=0}^{k}\left(j \alpha_{j}-\beta_{j}\right), \\
C_{2} & =\sum_{j=0}^{k}\left(\frac{j^{2}}{2 !} \alpha_{j}-j \beta_{j}-\gamma_{j}\right), \\
& \vdots \\
C_{q} & =\sum_{j=0}^{k}\left(\frac{j^{q}}{q !} \alpha_{j}-\frac{j^{q-1}}{(q-1) !} \beta_{j}-\frac{j^{q-2}}{(q-2) !} \gamma_{j}\right), \quad \text { where } q=3,4,5, \ldots
\end{aligned}
$$

Therefore, the order and error constant of the two-point one-step block method can be obtained by using equation in (2.8). 
For $q=0$,

$$
C_{0}=\sum_{j=0}^{3} \frac{1}{0 !} j^{0} \alpha_{j}=\alpha_{0}+\alpha_{1}+\alpha_{2}+\alpha_{3}=\left[\begin{array}{l}
0 \\
0 \\
0 \\
0
\end{array}\right]+\left[\begin{array}{c}
0 \\
-1 \\
0 \\
0
\end{array}\right]+\left[\begin{array}{c}
0 \\
1 \\
0 \\
-1
\end{array}\right]+\left[\begin{array}{l}
0 \\
0 \\
0 \\
1
\end{array}\right]=\left[\begin{array}{l}
0 \\
0 \\
0 \\
0
\end{array}\right] .
$$

For $q=1$,

$$
\begin{aligned}
C_{1} & =\sum_{j=0}^{3} \frac{1}{1 !} j^{1} \alpha_{j}-\sum_{j=0}^{3} \frac{1}{0 !} j^{0} \beta_{j} \\
& =1^{1} \cdot \alpha_{1}+2^{1} \cdot \alpha_{2}+3^{1} \cdot \alpha_{3}-\left(\beta_{0}+\beta_{1}+\beta_{2}+\beta_{3}\right) \\
& =1\left[\begin{array}{c}
0 \\
-1 \\
0 \\
0
\end{array}\right]+2\left[\begin{array}{c}
0 \\
1 \\
0 \\
-1
\end{array}\right]+3\left[\begin{array}{l}
0 \\
0 \\
0 \\
1
\end{array}\right]-\left(\left[\begin{array}{l}
0 \\
0 \\
0 \\
0
\end{array}\right]+\left[\begin{array}{l}
1 \\
1 \\
0 \\
0
\end{array}\right]+\left[\begin{array}{c}
-1 \\
0 \\
1 \\
1
\end{array}\right]+\left[\begin{array}{c}
0 \\
0 \\
-1 \\
0
\end{array}\right]\right)=\left[\begin{array}{l}
0 \\
0 \\
0 \\
0
\end{array}\right] .
\end{aligned}
$$

For $q=2$,

$$
\begin{aligned}
C_{2}= & \sum_{j=0}^{3} \frac{1}{2 !} j^{2} \alpha_{j}-\sum_{j=0}^{3} \frac{1}{1 !} j^{1} \beta_{j}-\sum_{j=0}^{3} \frac{1}{0 !} j^{0} \gamma_{j} \\
= & \frac{1}{2}\left(1^{2} \cdot \alpha_{1}+2^{2} \cdot \alpha_{2}+3^{2} \cdot \alpha_{3}\right)-\left(1^{1} \cdot \beta_{1}+2^{1} \cdot \beta_{2}+3^{1} \cdot \beta_{3}\right) \\
& -\left(\gamma_{0}+\gamma_{1}+\gamma_{2}+\gamma_{3}\right) \\
= & \left.\frac{1}{2}\left(\left[\begin{array}{l}
0 \\
-1 \\
0 \\
0
\end{array}\right]+4\left[\begin{array}{l}
0 \\
1 \\
0 \\
-1
\end{array}\right]+9\left[\begin{array}{l}
0 \\
0 \\
0 \\
1
\end{array}\right]\right)-\left(\begin{array}{l}
1 \\
1 \\
0 \\
0
\end{array}\right]+2\left[\begin{array}{c}
1 \\
0 \\
1 \\
1
\end{array}\right]+3\left[\begin{array}{c}
-1 \\
0 \\
-1 \\
0
\end{array}\right]\right) \\
& \left.-\left[\begin{array}{l}
0 \\
0 \\
0 \\
0
\end{array}\right]+\left[\begin{array}{c}
0 \\
\frac{7}{24} \\
\frac{-1}{12} \\
\frac{-1}{24}
\end{array}\right]+\left[\begin{array}{c}
\frac{8}{12} \\
\frac{6}{24} \\
\frac{8}{12} \\
\frac{10}{24}
\end{array}\right]+\left[\begin{array}{c}
\frac{-1}{12} \\
\frac{-1}{24} \\
\frac{5}{12} \\
\frac{3}{24}
\end{array}\right]\right)\left[\begin{array}{l}
0 \\
0 \\
0 \\
0
\end{array}\right] .
\end{aligned}
$$


For $q=3$,

$$
C_{3}=\sum_{j=0}^{3} \frac{1}{3 !} j^{3} \alpha_{j}-\sum_{j=0}^{3} \frac{1}{2 !} j^{2} \beta_{j}-\sum_{j=0}^{3} \frac{1}{1 !} j^{1} \gamma_{j}=\left[\begin{array}{l}
0 \\
0 \\
0 \\
0
\end{array}\right]
$$

For $q=4$,

$$
C_{4}=\sum_{j=0}^{3} \frac{1}{4 !} j^{4} \alpha_{j}-\sum_{j=0}^{3} \frac{1}{3 !} j^{3} \beta_{j}-\sum_{j=0}^{3} \frac{1}{2 !} j^{2} \gamma_{j}=\left[\begin{array}{l}
0 \\
0 \\
0 \\
0
\end{array}\right]
$$

For $q=5$,

$$
C_{5}=\sum_{j=0}^{3} \frac{1}{5 !} j^{5} \alpha_{j}-\sum_{j=0}^{3} \frac{1}{4 !} j^{4} \beta_{j}-\sum_{j=0}^{3} \frac{1}{3 !} j^{3} \gamma_{j}=\left[\begin{array}{c}
\frac{1}{24} \\
\frac{1}{45} \\
-\frac{1}{24} \\
-\frac{7}{360}
\end{array}\right] \neq\left[\begin{array}{l}
0 \\
0 \\
0 \\
0
\end{array}\right] .
$$

The method is order $p$ if $C_{0}=C_{1}=\cdots=C_{p+1}=0$ and $C_{p+2} \neq 0$ is the error constant. Thus, we conclude that the method in (2.3) and (2.4) is of the order 3 and the error constant is

$$
C_{p+2}=C_{5}=\left[\frac{1}{24}, \frac{1}{45},-\frac{1}{24},-\frac{7}{360}\right]^{\mathrm{T}} .
$$

\section{Implementation of the Method}

The initial starting point at each block are obtained by using Euler method as predictor and the initial $h$ used is as follows:

$$
h_{\text {initial }}=\left(\frac{\mathrm{TOL}}{2}\right)^{1 /(p+1)}
$$

where TOL is the tolerance and $p$ is the order of the method. Then, the calculations are corrected using the corrector formulae in (2.3) and (2.4). For the next block, the 
same techniques are repeated to compute the approximation values of $y_{n+1}$ and $y_{n+2}$ simultaneously until the end of the interval. We use $r$ function evaluations per step at the corrector formulae, that is, $P E(C E)^{r}$, where $P, E$, and $C$ indicate the predictor, evaluate the function $f$, and the corrector, respectively.

Algorithm 3.1. Computing approximating $y_{n+1,0^{\prime}}^{\prime(p)} y_{n+1,0^{\prime}}^{(p)} y_{n+2,0^{\prime}}^{\prime(p)}$ and $y_{n+2,0}^{(p)}$ using the predictor formulas is as follows:

$$
\begin{aligned}
& \text { for } i=1 \text { to } 2 \text { do } \\
& \qquad \begin{aligned}
P: y_{n+i, 0}^{\prime(p)}=y_{n+(i-1)}^{\prime(p)}+h f_{n+(i-1)^{\prime}}^{(p)} \\
y_{n+i, 0}^{(p)}=y_{n+(i-1)}^{(p)}+h y_{n+(i-1)}^{\prime(p)}+\frac{h^{2}}{2} f_{n+(i-1)}^{(p)}, \\
E: f\left(x_{n+i}, y_{n+i, 0}^{(p)}, y_{n+i, 0}^{\prime(p)}\right)
\end{aligned}
\end{aligned}
$$

\section{end for}

Computing approximations $y_{n+1,0}^{\prime(c)}, y_{n+1,0}^{(c)}, y_{n+2,0^{\prime}}^{\prime(c)}$ and $y_{n+2,0}^{(c)}$ using corrector formulas is as follows:

$$
\begin{aligned}
& \text { for } r=0 \text { do } \\
& \qquad \begin{aligned}
C: & y_{n+1, r+1}^{(c)}=y_{n}^{\prime(c)}+\frac{h}{12}\left(5 f_{n}^{(c)}+8 f_{n+1,0}^{(p)}-f_{n+2,0}^{(p)}\right), \\
& y_{n+1, r+1}^{(c)}=y_{n}^{(c)}+h y_{n}^{\prime(c)}+\frac{h^{2}}{24}\left(7 f_{n}^{(c)}+6 f_{n+1,0}^{(p)}-f_{n+2,0}^{(p)}\right), \\
& y_{n+2, r+1}^{\prime(c)}=y_{n+1, r+1}^{\prime(c)}+\frac{h}{12}\left(-f_{n}^{(c)}+8 f_{n+1,0}^{(p)}+5 f_{n+2,0}^{(p)}\right), \\
& y_{n+2, r+1}^{(c)}=y_{n+1, r+1}^{(c)}+h y_{n+1, r+1}^{\prime(c)}+\frac{h^{2}}{24}\left(-f_{n}^{(c)}+10 f_{n+1,0}^{(p)}+3 f_{n+2,0}^{(p)}\right),
\end{aligned}
\end{aligned}
$$

for $i=1$ to 2 do

$$
E: f\left(x_{n+i}, y_{n+i, r+1}^{(c)}, y_{n+i, r+1}^{\prime(c)}\right)
$$

\section{end for}

\section{end for}

for $r=1,2, \ldots$ until convergence do 


$$
\begin{aligned}
C: y_{n+1, r+1}^{\prime(c)} & =y_{n}^{\prime(c)}+\frac{h}{12}\left(5 f_{n}^{(c)}+8 f_{n+1,0}^{(c)}-f_{n+2,0}^{(c)}\right), \\
y_{n+1, r+1}^{(c)} & =y_{n}^{(c)}+h y_{n}^{\prime(c)}+\frac{h^{2}}{24}\left(7 f_{n}^{(c)}+6 f_{n+1,0}^{(c)}-f_{n+2,0}^{(c)}\right), \\
y_{n+2, r+1}^{\prime(c)} & =y_{n+1, r+1}^{\prime(c)}+\frac{h}{12}\left(-f_{n}^{(c)}+8 f_{n+1,0}^{(c)}+5 f_{n+2,0}^{(c)}\right), \\
y_{n+2, r+1}^{(c)} & =y_{n+1, r+1}^{(c)}+h y_{n+1, r+1}^{\prime(c)}+\frac{h^{2}}{24}\left(-f_{n}^{(c)}+10 f_{n+1,0}^{(c)}+3 f_{n+2,0}^{(c)}\right),
\end{aligned}
$$

for $i=1$ to 2 do

$$
E: f\left(x_{n+i}, y_{n+i, r+1}^{(c)}, y_{n+i, r+1}^{\prime(c)}\right)
$$

end for

\section{end for}

The convergence test:

$$
\left|\frac{\left(y_{n+2, r+1}\right)_{t}-\left(y_{n+2, r}\right)_{t}}{A+B\left(y_{n+2, r+1}\right)_{t}}\right|<0.1 \times \mathrm{TOL}
$$

where $r$ is the number of iterations and $(y)_{t}$ is the $t$ th component of the approximate. $A=1$, $B=0$ correspond to the absolute error test. $A=1, B=1$ correspond to the mixed test and finally $A=0, B=1$ correspond to the relative error test. The mixed error test is used in all tested problems. If the convergence test is satisfied, then we control the error in the current block by performing the local truncation error as follows:

$$
\mathrm{LTE}=\left|y_{n+2}^{(k)}-y_{n+2}^{(k-1)}\right|
$$

where $k$ is the order of corrector formula. The errors calculated in the code is defined as

$$
\left(E_{i}\right)_{t}=\left|\frac{\left(y_{i}\right)_{t}-\left(y\left(x_{i}\right)\right)_{t}}{A+B\left(y\left(x_{i}\right)\right)_{t}}\right| .
$$

For the evaluation of maximum error, it is defined as follow:

$$
\operatorname{MAXE}=\max _{1 \leq i \leq \mathrm{TS}}\left(\max _{1 \leq i \leq N}\left(E_{i}\right)_{t}\right),
$$

where $N$ is the number of equation in the system and TS is the number of successful steps. 
In order to make the selection of the next step size, we follow again the techniques used in [9]. If LTE $\leq$ TOL, then the next step size remains constant or double, otherwise the step size will be half. The step size when the integration steps are successful is given by

$$
\begin{aligned}
& h_{\text {new }}=C \times h_{\text {old }} \times\left(\frac{\text { TOL }}{\mathrm{LTE}}\right)^{1 /(k+1)}, \\
& \text { if }\left(h_{\text {new }} \geq 2 \times h_{\text {old }}\right) \text {, then } h_{\text {next }}=2 \times h_{\text {old }}, \\
& \text { else } h_{\text {next }}=h_{\text {old }},
\end{aligned}
$$

where $C=0.5$ is a safety factor. The algorithm when the step failure occurs is

$$
h_{\text {next }}=\frac{1}{2} \times h_{\text {old }}
$$

\section{Stability of the Method}

In this section, we will discuss the stability of the proposed method derived in the previous section on a linear general second-order problem:

$$
y^{\prime \prime}=f=\theta y^{\prime}+\lambda y
$$

Firstly, the test equation in (4.1) is substituted into the predictor formula (3.2). The evaluation of $f_{n+1,0}^{p}$ and $f_{n+2,0}^{p}$ will be substituted into the right-hand side of (3.3) when $r=0$ as shown below:

$$
\begin{aligned}
& f_{n+1,0}^{p}=\theta y_{n+1,0}^{\prime p}+\lambda y_{n+1,0}^{p} \\
& f_{n+2,0}^{p}=\theta y_{n+2,0}^{\prime p}+\lambda y_{n+2,0^{\prime}}^{p}
\end{aligned}
$$

where $y_{n+1,0}^{\prime p}, y_{n+1,0}^{p} y_{n+2,0^{\prime}}^{\prime p}$ and $y_{n+2,0}^{p}$ in (4.2) are obtained in (3.2) after substituted the test equation (4.1). Then, the formulae are written in the matrix form and setting the determinant of the matrix to zero. Hence, the stability polynomial is obtained:

$$
\begin{aligned}
& r=0: \\
& t^{2}\left(1+\frac{25 h \theta}{12}+\frac{9 h^{2} \theta^{2}}{4}+\frac{5 h^{3} \theta^{3}}{6}+\frac{h^{4} \lambda^{4}}{3}+\frac{h^{2} \lambda}{8}-\frac{h^{3} \theta \lambda}{2}+\frac{h^{4} \theta^{2} \lambda}{12}\right. \\
& \left.\quad-\frac{h^{5} \theta^{3} \lambda}{12}-\frac{37 h^{4} \lambda^{2}}{144}-\frac{h^{5} \theta \lambda^{2}}{9}-\frac{h^{6} \theta^{2} \lambda^{2}}{9}-\frac{h^{6} \lambda^{3}}{36}+\frac{h^{7} \theta \lambda^{3}}{16}+\frac{h^{8} \lambda^{4}}{36}\right)
\end{aligned}
$$




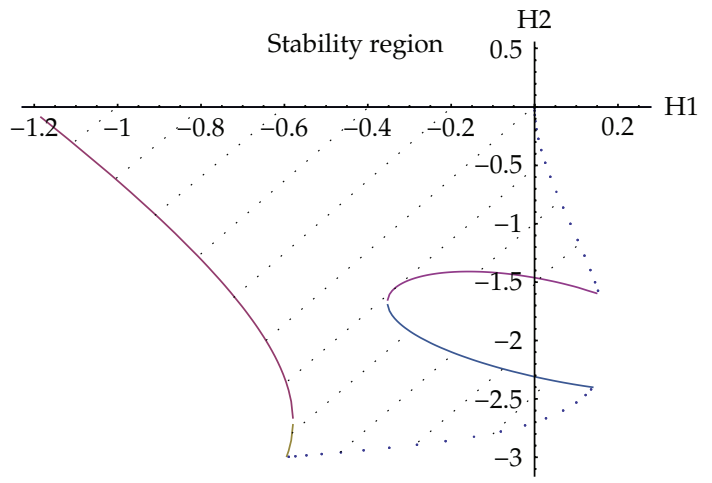

Figure 2: Stability region for direct block one-step method when $r=0$.

$$
\begin{aligned}
& +t^{3}\left(-2-\frac{13 h \theta}{6}-\frac{7 h^{2} \theta^{2}}{3}-\frac{5 h^{3} \theta^{3}}{6}-\frac{h^{4} \lambda^{4}}{3}-\frac{17 h^{2} \lambda}{4}-\frac{47 h^{3} \theta \lambda}{12}-\frac{7 h^{4} \theta^{2} \lambda}{4}\right. \\
& \left.\quad-\frac{7 h^{5} \theta^{3} \lambda}{12}-\frac{115 h^{4} \lambda^{2}}{72}-\frac{19 h^{5} \theta \lambda^{2}}{18}-\frac{7 h^{6} \theta^{2} \lambda^{2}}{18}-\frac{7 h^{6} \lambda^{3}}{36}-\frac{17 h^{7} \theta \lambda^{3}}{144}-\frac{h^{8} \lambda^{4}}{72}\right) \\
& +t^{4}\left(1+\frac{h \theta}{12}+\frac{h^{2} \theta^{2}}{12}+\frac{h^{2} \lambda}{8}+\frac{h^{3} \theta \lambda}{12}+\frac{h^{4} \lambda^{2}}{48}\right)=0 .
\end{aligned}
$$

Figure 2 showed the stability region of the direct block one-step method when $r=0$. In case $r=1$, the stability polynomial is obtained as follows:

$$
\begin{aligned}
& r=1: \\
& t^{2}\left(1+2 h \theta+\frac{25 h^{2} \theta^{2}}{12}+\frac{19 h^{3} \theta^{3}}{12}+\frac{h^{4} \theta^{4}}{3}+\frac{5 h^{5} \theta^{5}}{18}+\frac{h^{6} \theta^{6}}{9}+\frac{7}{48} h^{3} \theta \lambda-\frac{13}{48} h^{4} \theta^{2} \lambda+\frac{1}{4} h^{5} \theta^{3} \lambda\right. \\
& +\frac{17}{72} h^{6} \theta^{4} \lambda+\frac{1}{18} h^{7} \theta^{5} \lambda+\frac{11 h^{4} \lambda^{2}}{288}-\frac{83}{144} h^{5} \theta \lambda^{2}+\frac{101}{432} h^{6} \theta^{2} \lambda^{2}+\frac{13}{432} h^{7} \theta^{3} \lambda^{2} \\
& -\frac{17}{432} h^{8} \theta^{4} \lambda^{2}-\frac{199 h^{6} \lambda^{3}}{1728}-\frac{53}{288} h^{7} \theta \lambda^{3}-\frac{7}{216} h^{8} \theta^{2} \lambda^{3}-\frac{5}{432} h^{9} \theta^{3} \lambda^{3} \\
& \left.-\frac{29 h^{8} \lambda^{4}}{324}-\frac{17 h^{9} \theta \lambda^{4}}{1296}+\frac{97 h^{10} \theta^{2} \lambda^{4}}{5184}-\frac{h^{10} \lambda^{5}}{648}+\frac{1}{96} h^{11} \theta \lambda^{5}+\frac{h^{12} \lambda^{6}}{648}\right) \\
& +t^{3}\left(-2-2 h \theta-\frac{13 h^{2} \theta^{2}}{6}-\frac{5 h^{3} \theta^{3}}{3}-\frac{h^{4} \theta^{4}}{3}-\frac{5 h^{5} \theta^{5}}{18}-\frac{h^{6} \theta^{6}}{9}-4 h^{2} \lambda-\frac{103}{24} h^{3} \theta \lambda\right. \\
& \quad-\frac{17 h^{4} \theta^{2} \lambda}{6}-\frac{11 h^{5} \theta^{3} \lambda}{12}-\frac{19 h^{6} \theta^{4} \lambda}{24}-\frac{5 h^{7} \theta^{5} \lambda}{18}-\frac{203 h^{4} \lambda^{2}}{144}-\frac{199}{144} h^{5} \theta \lambda^{2}
\end{aligned}
$$




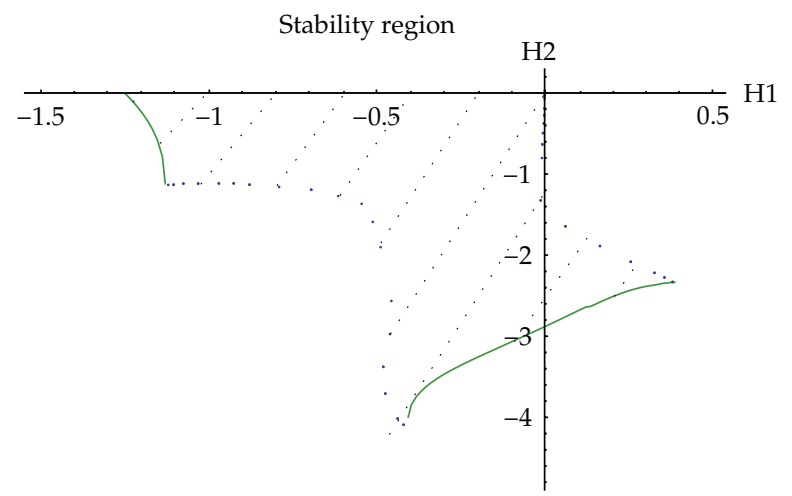

Figure 3: Stability region for direct block one-step method when $r=1$.

$$
\begin{gathered}
-\frac{365 h^{6} \theta^{2} \lambda^{2}}{432}-\frac{361}{432} h^{7} \theta^{3} \lambda^{2}-\frac{127 h^{8} \theta^{4} \lambda^{2}}{432}-\frac{133 h^{6} \lambda^{3}}{864}-\frac{269 h^{7} \theta \lambda^{3}}{864}-\frac{23 h^{8} \theta^{2} \lambda^{3}}{54} \\
\left.-\frac{73 h^{9} \theta^{3} \lambda^{3}}{432}-\frac{55 h^{8} \lambda^{4}}{1296}-\frac{139 h^{9} \theta \lambda^{4}}{1296}-\frac{289 h^{10} \theta^{2} \lambda^{4}}{5184}-\frac{7 h^{10} \lambda^{5}}{648}-\frac{13 h^{11} \theta \lambda^{5}}{1296}-\frac{h^{12} \lambda^{6}}{1296}\right) \\
+t^{4}\left(1+\frac{h^{2} \theta^{2}}{12}+\frac{h^{3} \theta^{3}}{12}+\frac{7}{48} h^{3} \theta \lambda+\frac{5}{48} h^{4} \theta^{2} \lambda+\frac{11 h^{4} \lambda^{2}}{288}+\frac{1}{24} h^{5} \theta \lambda^{2}+\frac{h^{6} \lambda^{3}}{192}\right)=0 .
\end{gathered}
$$

Figure 3 showed the stability region of the direct block one-step method when $r=1$.

The stability region is plotted using MATHEMATICA, and the shaded region inside the boundary in Figures 2 and 3 demonstrate the stability region for the proposed method.

\section{Numerical Results and Discussion}

We have tested the performance of the proposed method on four problems. For the first three problems, a comparison is made between the solutions obtained in [9].

Problem 1. We have

$$
\begin{array}{rr}
y_{1}^{\prime \prime}=-y_{2}+\sin \pi x, \quad y_{1}(0)=0, \quad y_{1}^{\prime}(0)=-1, \\
y_{2}^{\prime \prime}=-y_{1}+1-\pi^{2} \sin \pi x, \quad y_{2}(0)=1, \quad y_{2}^{\prime}(0)=1+\pi, \quad[0,10] .
\end{array}
$$

Solution: $y_{1}(x)=1-e^{x}, y_{2}(x)=e^{x}+\sin \pi x$.

First-order systems:

$$
\begin{gathered}
y_{1}^{\prime}=y_{3}, \quad y_{2}^{\prime}=y_{4}, \quad y_{3}^{\prime}=-y_{2}+\sin \pi x, \quad y_{4}^{\prime}=-y_{1}+1-\pi^{2} \sin \pi x, \\
y_{1}(0)=0, \quad y_{2}(0)=1, \quad y_{3}(0)=-1, \quad y_{4}(0)=1+\pi .
\end{gathered}
$$

Solution: $y_{1}(x)=1-e^{x}, y_{2}(x)=e^{x}+\sin \pi x, y_{3}(x)=-e^{x}, y_{4}(x)=e^{x}+\pi \cos \pi x$. 
Problem 2. We have

$$
\begin{gathered}
y_{1}^{\prime \prime}=-e^{-x} y_{2}, \quad y_{1}(0)=1, \quad y_{1}^{\prime}(0)=0, \\
y_{2}^{\prime \prime}=2 e^{x} y_{1}^{\prime}, \quad y_{2}(0)=1, \quad y_{2}^{\prime}(0)=1, \quad[0, \pi] .
\end{gathered}
$$

Solution: $y_{1}(x)=\cos x, y_{2}(x)=e^{x} \cos x$.

First-order systems:

$$
\begin{array}{lcc}
y_{1}^{\prime}=y_{3}, \quad y_{2}^{\prime}=y_{4}, \quad y_{3}^{\prime}=-e^{-x} y_{2}, \quad y_{4}^{\prime}=2 e^{x} y_{3}, \\
y_{1}(0)=1, \quad y_{2}(0)=1, \quad y_{3}(0)=0, \quad y_{4}(0)=1 .
\end{array}
$$

Solution: $y_{1}(x)=\cos x, y_{2}(x)=e^{x} \cos x, y_{3}(x)=-\sin x, y_{4}(x)=e^{x} \cos x-e^{x} \sin x$.

Problem 3. We have

$$
\begin{gathered}
y_{1}^{\prime \prime}=-y_{2}^{\prime}, \quad y_{1}(0)=0, \quad y_{1}^{\prime}(0)=\frac{1}{1-e^{-1}}, \\
y_{2}^{\prime \prime}=-y_{1}^{\prime}, \quad y_{2}(0)=1, \quad y_{2}^{\prime}(0)=\frac{1}{1-e^{-1}}, \quad[0,10]
\end{gathered}
$$

Solution: $y_{1}(x)=\left(1-e^{-x}\right) /\left(1-e^{-1}\right), y_{2}(x)=\left(2-e^{-1}-e^{-x}\right) /\left(1-e^{-1}\right)$.

First-order systems:

$$
\begin{gathered}
y_{1}^{\prime}=y_{3}, \quad y_{2}^{\prime}=y_{4}, \quad y_{3}^{\prime}=-y_{4}, \quad y_{4}^{\prime}=-y_{3}, \\
y_{1}(0)=0, \quad y_{2}(0)=1, \quad y_{3}(0)=\frac{1}{1-e^{-1}}, \quad y_{4}(0)=\frac{1}{1-e^{-1}} .
\end{gathered}
$$

Solution: $y_{1}(x)=\left(1-e^{-x}\right) /\left(1-e^{-1}\right), y_{2}(x)=\left(2-e^{-1}-e^{-x}\right) /\left(1-e^{-1}\right), y_{3}(x)=\left(e^{-x}\right) /\left(1-e^{-1}\right)$, $y_{4}(x)=e^{-x} /\left(1-e^{-1}\right)$.

Problem 4 (Van Der Pol oscillator). We have

$$
y^{\prime \prime}-2 \xi\left(1-y^{2}\right) y^{\prime}+y=0, \quad y(0)=0, \quad y^{\prime}(0)=0.5, \quad[0,10]
$$

where we take $\xi=0.025$.

The codes are written in C language and executed on DYNIX/ptx operating system. The numerical results for Problems 1-3 in Tables 2-4 are solved using the proposed method and the method in [9]. The results in terms of total steps and execution times for solving Problems 1-3 are presented in histograms and graph lines in Figures 4, 5 and 6 . The solutions of $2 \mathrm{P}(\mathrm{B})$ for solving Problem 4 are plotted in Figure 7 at tolerance $10^{-8}$ and compared with the solutions obtained by the MATLAB built-in solver ode45.

In Figures 4, 5 and 6, it is obvious that method $2 \mathrm{P}(\mathrm{B})$ requires less number of total steps as compared to method $2 \mathrm{P}(\mathrm{A})$ when solving the same given problems. It is also observed that 
Table 1: The ratios execution times and steps for solving Problems 1 to 3.

\begin{tabular}{lcccccc}
\hline \multirow{2}{*}{ TOL } & \multicolumn{2}{c}{ PROB 1 } & \multicolumn{2}{c}{ PROB 2 } & \multicolumn{2}{c}{ PROB 3 } \\
& RSTEP & RTIME & RSTEP & RTIME & RSTEP & RTIME \\
\hline $10^{-2}$ & 2.53 & 1.90 & 2.83 & 1.55 & 2.56 & 1.20 \\
$10^{-4}$ & 2.38 & 2.31 & 2.88 & 1.57 & 2.45 & 1.32 \\
$10^{-6}$ & 2.89 & 3.05 & 3.11 & 2.84 & 3.19 & 2.30 \\
$10^{-8}$ & 4.74 & 5.26 & 4.34 & 1.49 & 3.47 & 3.17 \\
\hline
\end{tabular}

Table 2: Comparison between $2 \mathrm{P}(\mathrm{A})$ and $2 \mathrm{P}(\mathrm{B})$ methods for solving Problem 1.

\begin{tabular}{lccccccc}
\hline TOL & Method & TS & FS & MAXE & AVERR & FCN & TIME \\
\hline \multirow{2}{*}{$10^{-2}$} & $2 \mathrm{P}(\mathrm{A})$ & 48 & 2 & $3.20591 e-004$ & $1.28467 e-004$ & 385 & 1002 \\
& $2 \mathrm{P}(\mathrm{B})$ & 19 & 0 & $3.88324 e-003$ & $2.89565 e-003$ & 121 & 527 \\
\hline \multirow{2}{*}{$10^{-4}$} & $2 \mathrm{P}(\mathrm{A})$ & 143 & 2 & $3.69289 e-006$ & $1.48547 e-006$ & 1145 & 2423 \\
& $2 \mathrm{P}(\mathrm{B})$ & 60 & 0 & $3.46171 e-005$ & $2.81402 e-005$ & 367 & 1047 \\
\hline \multirow{2}{*}{$10^{-6}$} & $2 \mathrm{P}(\mathrm{A})$ & 546 & 3 & $2.91502 e-008$ & $7.74544 e-009$ & 4369 & 9287 \\
& $2 \mathrm{P}(\mathrm{B})$ & 189 & 0 & $3.43754 e-007$ & $2.76828 e-007$ & 1133 & 3045 \\
\hline \multirow{2}{*}{$10^{-8}$} & $2 \mathrm{P}(\mathrm{A})$ & 2819 & 3 & $2.43829 e-011$ & $9.79663 e-012$ & 22553 & 47821 \\
& $2 \mathrm{P}(\mathrm{B})$ & 595 & 0 & $3.44148 e-009$ & $2.75811 e-009$ & 3571 & 9093 \\
\hline
\end{tabular}

the execution times of $2 \mathrm{P}(\mathrm{B})$ are faster than $2 \mathrm{P}(\mathrm{A})$ at all tested tolerances. This is expected since $2 \mathrm{P}(\mathrm{B})$ has less number of function calls; therefore, it has affected the computation time of $2 \mathrm{P}(\mathrm{B})$.

In Table 1, the RSTEP and RTIME are greater than 1.00 which shows that $2 \mathrm{P}(\mathrm{B})$ is more efficient compared to $2 \mathrm{P}(\mathrm{A})$. In fact, in some cases, the ratios are greater than 3.00 , which indicates a clear advantage of method $2 \mathrm{P}(\mathrm{B})$ over $2 \mathrm{P}(\mathrm{A})$.

In Table 2, it can be observed that the maximum error of $2 \mathrm{P}(\mathrm{B})$ is one or two order larger than $2 \mathrm{P}(\mathrm{A})$ but still acceptable as it is within the given tolerance. This is expected since the code $2 \mathrm{P}(\mathrm{B})$ solved the given problem directly without reducing to system of first-order differential equations. We could observe that the RSTEP for Problem 1 is greater than 2 in Table 1.

In Tables 3 and 4 , it is observed that the maximum error of $2 \mathrm{P}(\mathrm{B})$ is two or three order larger as compared to $2 \mathrm{P}(\mathrm{A})$ in solving Problems 2 and 3 but it is still within the given tolerances. In Figure 7, it is obvious that the $2 \mathrm{P}(\mathrm{B})$ solutions agree very well with the solutions obtained by the MATLAB built-in solver ode45 when solving Problem 4 .

\section{Conclusion}

In this paper, we have constructed the direct two-point block one-step method which is efficient and suitable for solving general second-order ODEs directly. The block method has shown acceptable solutions and managed to solve the second-order ODE faster compared to the existing method. 


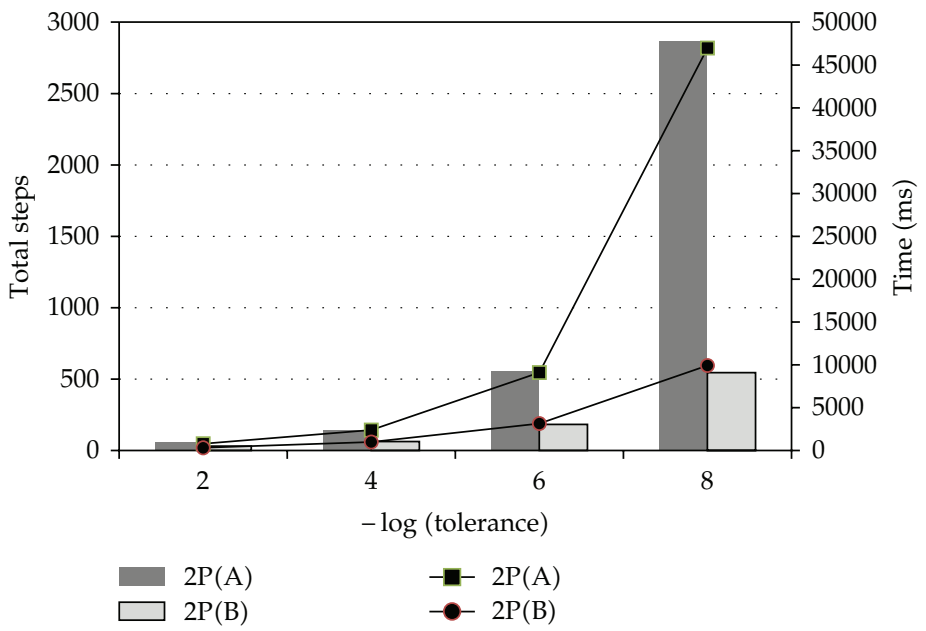

Figure 4: Results of total steps and time for Problem 1.

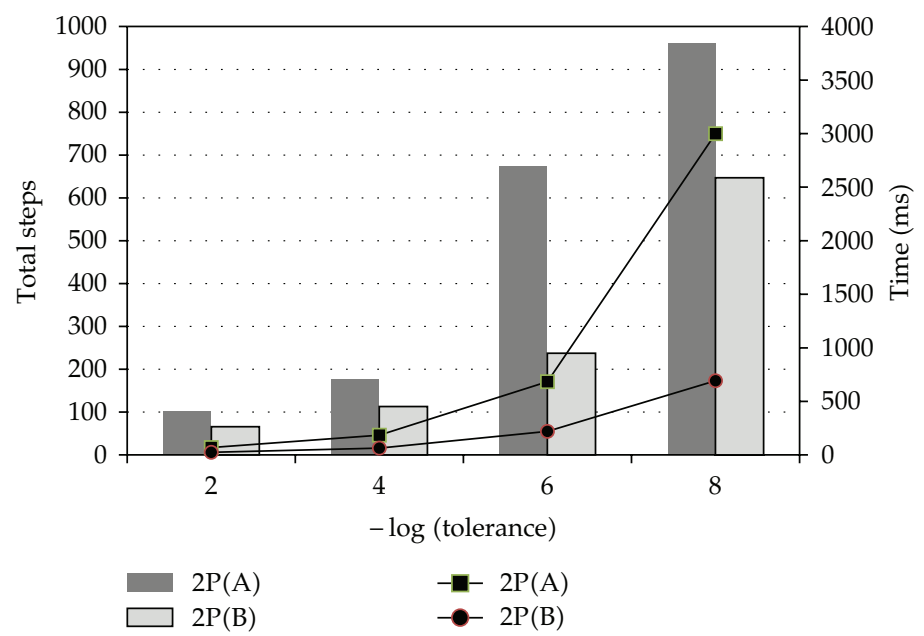

Figure 5: Results of total steps and time for Problem 2.

Table 3: Comparison between $2 \mathrm{P}(\mathrm{A})$ and 2P(B) methods for solving Problem 2.

\begin{tabular}{lccccccc}
\hline TOL & Method & TS & FS & MAXE & AVERR & FCN & TIME \\
\hline \multirow{2}{*}{$10^{-2}$} & $2 \mathrm{P}(\mathrm{A})$ & 17 & 4 & $4.64239 e-004$ & $1.01900 e-004$ & 137 & 408 \\
& 2P(B) & 6 & 0 & $1.29794 e-003$ & $2.48275 e-004$ & 47 & 264 \\
\hline \multirow{2}{*}{$10^{-4}$} & $2 \mathrm{P}(\mathrm{A})$ & 46 & 3 & $5.27751 e-006$ & $9.23287 e-007$ & 369 & 708 \\
& $2 \mathrm{P}(\mathrm{B})$ & 16 & 1 & $4.19030 e-005$ & $7.96402 e-006$ & 131 & 452 \\
\hline \multirow{2}{*}{$10^{-6}$} & $2 \mathrm{P}(\mathrm{A})$ & 171 & 2 & $2.35992 e-008$ & $5.04232 e-009$ & 1369 & 2694 \\
& $2 \mathrm{P}(\mathrm{B})$ & 55 & 1 & $4.24624 e-007$ & $6.60118 e-008$ & 429 & 950 \\
\hline \multirow{2}{*}{$10^{-8}$} & $2 \mathrm{P}(\mathrm{A})$ & 750 & 2 & $4.50146 e-011$ & $7.04651 e-012$ & 1741 & 3844 \\
& $2 \mathrm{P}(\mathrm{B})$ & 173 & 1 & $4.31085 e-009$ & $6.26858 e-010$ & 1343 & 2588 \\
\hline
\end{tabular}




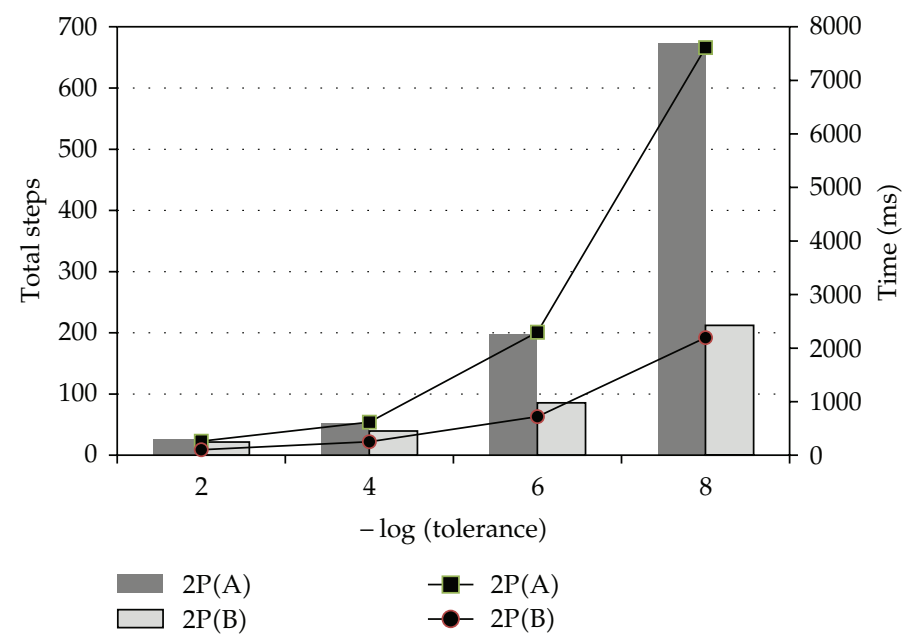

Figure 6: Results of total steps and time for Problem 3.

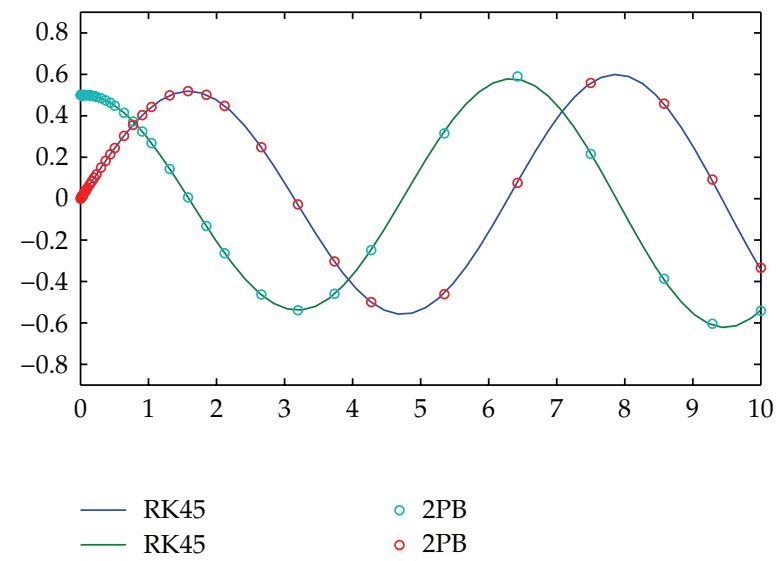

Figure 7: Solution of Problem 4 when TOL $=10^{-8}$.

Table 4: Comparison between $2 \mathrm{P}(\mathrm{A})$ and $2 \mathrm{P}(\mathrm{B})$ methods for solving Problem 3.

\begin{tabular}{lccccccc}
\hline TOL & Method & TS & FS & MAXE & AVERR & FCN & TIME \\
\hline \multirow{2}{*}{$10^{-2}$} & $2 \mathrm{P}(\mathrm{A})$ & 23 & 7 & $1.42058 e-004$ & $3.37359 e-005$ & 185 & 296 \\
& $2 \mathrm{P}(\mathrm{B})$ & 9 & 0 & $1.47268 e-003$ & $4.24039 e-004$ & 69 & 247 \\
\hline \multirow{2}{*}{$10^{-4}$} & $2 \mathrm{P}(\mathrm{A})$ & 54 & 2 & $1.41231 e-006$ & $2.21945 e-007$ & 433 & 597 \\
& $2 \mathrm{P}(\mathrm{B})$ & 22 & 0 & $2.73310 e-005$ & $9.95954 e-006$ & 177 & 451 \\
\hline \multirow{2}{*}{$10^{-6}$} & $2 \mathrm{P}(\mathrm{A})$ & 201 & 1 & $6.04402 e-009$ & $9.73203 e-010$ & 1609 & 2252 \\
& $2 \mathrm{P}(\mathrm{B})$ & 63 & 0 & $9.51564 e-007$ & $2.58666 e-007$ & 503 & 980 \\
\hline \multirow{2}{*}{$10^{-8}$} & $2 \mathrm{P}(\mathrm{A})$ & 666 & 1 & $4.12406 e-011$ & $8.11194 e-012$ & 5329 & 7700 \\
& $2 \mathrm{P}(\mathrm{B})$ & 192 & 0 & $1.25592 e-008$ & $2.60498 e-009$ & 1537 & 2426 \\
\hline
\end{tabular}




\section{Notations}

TOL: Tolerance

MTD: Method employed

TS: $\quad$ Total steps taken

FS: $\quad$ Total failure steps

MAXE: Magnitude of the maximum error of the computed solution

AVERR: The average error

FCN: Total function calls

TIME: The execution time taken in microseconds

2P(A): Implementation of the direct block method in [9] by reducing the problem to system of first-order ODEs

2P(B): Implementation of the direct two-point block one-step method by solving the problem directly

RSTEP: The ratio steps, $\mathrm{TS}_{2 \mathrm{P}(\mathrm{A})} / \mathrm{TS}_{2 \mathrm{P}(\mathrm{B})}$

RTIME: The ratio execution times, $\mathrm{TIME}_{2 \mathrm{P}(\mathrm{A})} / \mathrm{TIME}_{2 \mathrm{P}(\mathrm{B})}$

\section{Acknowledgment}

The authors would like to thank the Universiti Putra Malaysia for providing financial support through Graduate Research Fellowship (GRF) during the study period.

\section{References}

[1] J. B. Rosser, "A Runge-Kutta for all seasons," SIAM Review, vol. 9, pp. 417-452, 1967.

[2] L. F. Shampine and H. A. Watts, "Block implicit one-step methods," Mathematics of Computation, vol. 23, pp. 731-740, 1969.

[3] P. B. Worland, "Parallel methods for the numerical solution of ordinary differential equations," Institute of Electrical and Electronics Engineers, vol. 25, no. 10, pp. 1045-1048, 1976.

[4] S. Mehrkanoon, Z. A. Majid, and M. Suleiman, "A variable step implicit block multistep method for solving first-order ODEs," Journal of Computational and Applied Mathematics, vol. 233, no. 9, pp. 23872394, 2010.

[5] Z. A. Majid and M. B. Suleiman, "Implementation of four-point fully implicit block method for solving ordinary differential equations," Applied Mathematics and Computation, vol. 184, no. 2, pp. 514$522,2007$.

[6] Z. A. Majid, N. A. Azmi, and M. Suleiman, "Solving second order ordinary differential equations using two point four step direct implicit block method," European Journal of Scientific Research, vol. 31, no. 1, pp. 29-36, 2009.

[7] Z. B. Ibrahim, M. B. Suleiman, and F. Ismail, "Fully implicit two point block backward difference formula for solving a first order initial value problems," Science Putra Research Bulletin, vol. 11, no. 2, pp. 14-17, 2003.

[8] P. C. Chakravarti and P. B. Worland, "A class of self-starting methods for the numerical solution of $y^{\prime \prime}=f(x, y), "$ BIT, vol. 11, pp. 368-383, 1971.

[9] Z. A. Majid, M. B. Suleiman, F. Ismail, and M. Othman, "2-point implicit block one-step method half Gauss-Seidel for solving first order ordinary differential equations," Matematika, vol. 19, pp. 91-100, 2003.

[10] S. O. Fatunla, "Block methods for second order ODEs," International Journal of Computer Mathematics, vol. 41, pp. 55-63, 1991.

[11] J. D. Lambert, Computational Methods in Ordinary Differential Equations, John Wiley \& Sons, New York, NY, USA, 1973.

[12] C. W. Gear, Numerical Initial Value Problems in Ordinary Differential Equations, Prentice-Hall, Englewood Cliffs, NJ, USA, 1971. 


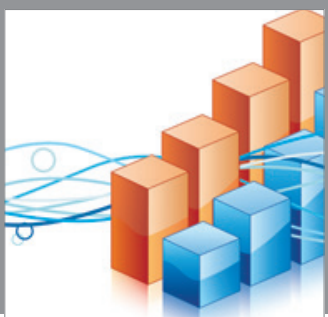

Advances in

Operations Research

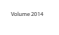

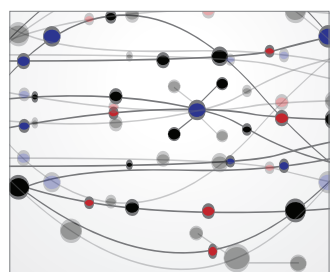

\section{The Scientific} World Journal
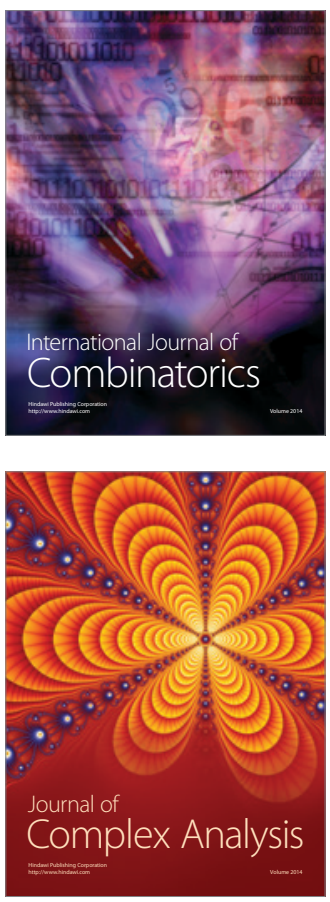

International Journal of

Mathematics and

Mathematical

Sciences
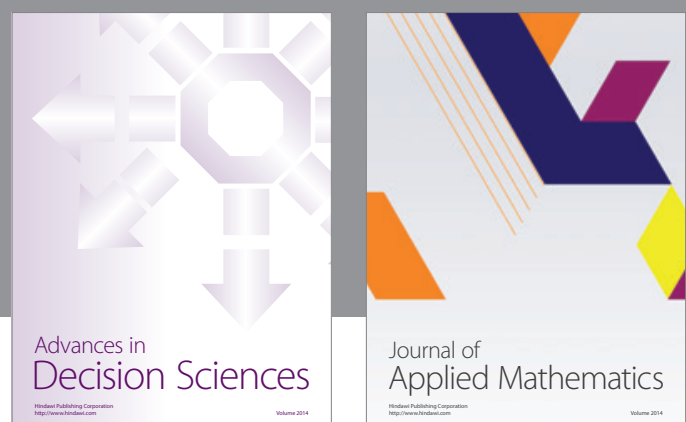

Journal of

Applied Mathematics
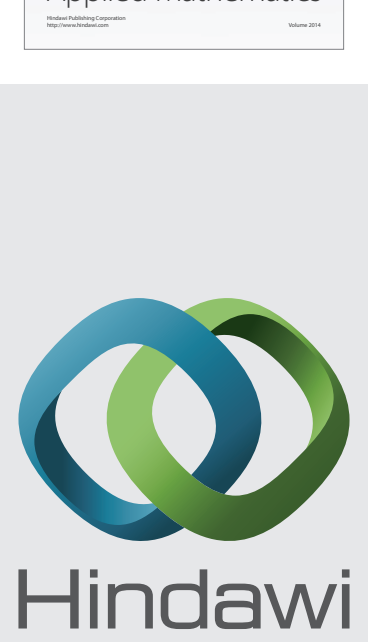

Submit your manuscripts at http://www.hindawi.com
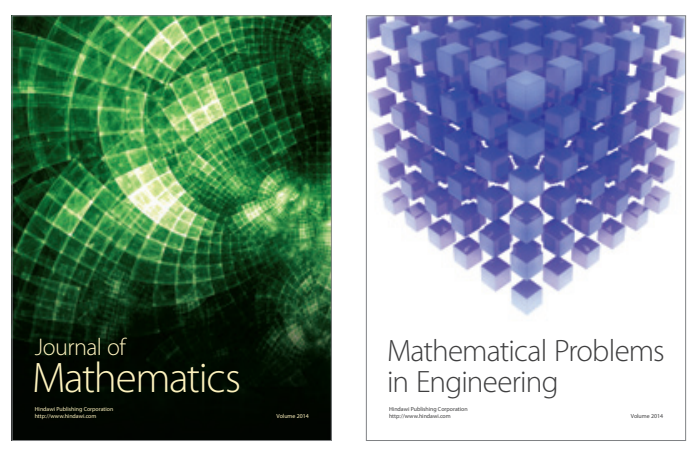

Mathematical Problems in Engineering
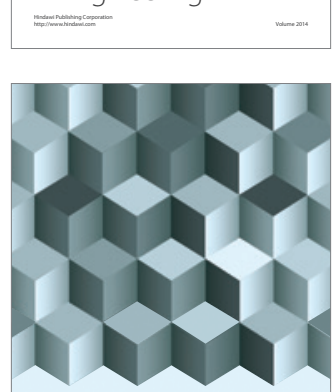

Journal of

Function Spaces
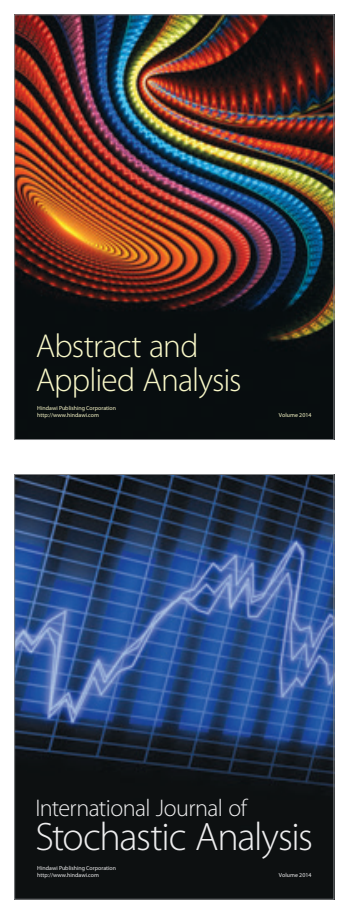

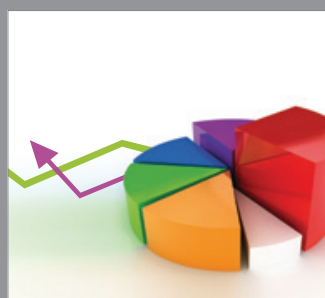

ournal of

Probability and Statistics

Promensencen
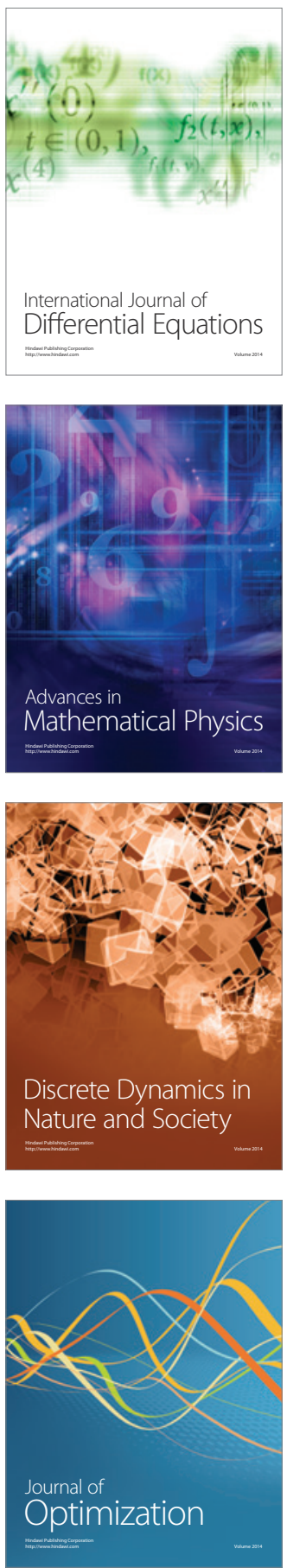